\title{
UCRL-JC-114999
}

PREPRINT

\section{Characterization of Defect Geometries in Multilayer Optical Coatings}

\author{
R. Tench, R. Chow and M. R. Kozlowski
}

This paper was prepared for submittal to the Laser-Induced Damage in Optical Materials October 27-29, 1993

Boulder, Colorado

\section{December 1993}

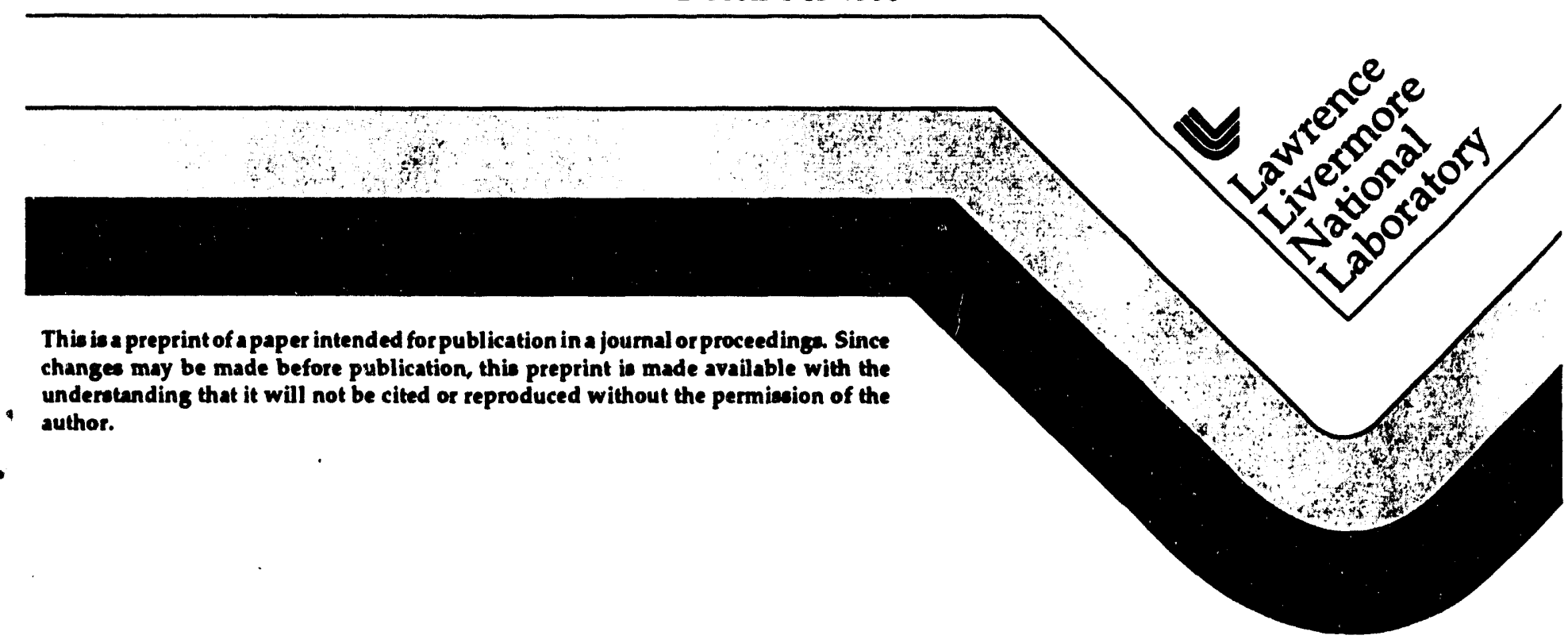




\section{DISCI.AIMER}

This document was prepared as an account of work sponsored by an agency of the United States Government. Neither the United States Government nor the University of California nor any of their employees, makes any warranty, express or implied, or assumes any legal liability or responsibility for the accuracy, completeness, or usefulness of any information, apparatus, product, or process disclosed, or represents that its use would not infringe privately owned rights. Reference herein to any specific commercial products, process, or service by trade name, trademark, manufacturer, or otherwise, does not necessarily constitute or imply its endorsement, recommendation. or favoring by the United States Government or the Liniversity of California. The viens and opinions of authors expressed herein do not necessarily state or reflect those of the United States Government or the University of California, and shall not be used for advertising or product ensorsement purposes. 


\title{
Characterization of defect geometries \\ in multilayer optical coatings
}

\author{
R. J. Tench, R. Chow, and M. R. Kozlowski \\ Lawrence Livermore National Laboratory \\ P. O. Box 808 , L-340 \\ Livermore, CA 94551
}

\begin{abstract}
Laser-induced damage in optical coatings is generally associated with micrometer-scale defects. A simple geometric model for nodule-shaped defects is commonly used to describe defects in optical coatings. No systematic study has been done, however, to prove the applicability of that model to standard optical coating deposition. Some defects are known not to have a classic nodule geometry. The present study uses atomic force microscopy (AFM) and scanning electron microscopy to characterize the topography of coating defects in a $\mathrm{HfO}_{2} / \mathrm{SiO}_{2}$ multilayer mirror system. Focused ionbeam cross-sectioning is then used to study the underlying defect structure. This work develops a model for defect shape such that the overall geometry of a coating defect, particularly seed size and depth, can be inferred from non-destructive evaluation measurements such as AFM. The relative mechanical stabilities of nodular defects can be deduced based on the nodule's geometry. Auger analysis showed that the seed material that causes nodular defects in $\mathrm{HfO}_{2} / \mathrm{SiO}_{2}$ multilayers is a hafnia oxide. Such characterization capabilities are needed for understanding the enhanced susceptibility of particular defecis to laser damage and for developing improved techniques for depositing low-defect density coatings.
\end{abstract}

\section{INTRODUCTION}

High power laser systems currently under design are limited by the laser-induced damage thresholds of multilayer optical coatings used as mirrors and polarizers. It has generally been accepted that the low damage threshold is associated with nodular defects in the coatings. 1,2,3 It is assumed that most of these nodular defects are caused by shadowing effects initiated by seed particles during the deposition process. ${ }^{1,2,4}$ Not only are these defects associated with the damage threshold, there is also strong evidence that the laser conditioning effect also influences defects. ${ }^{5}$

In general, it has been observed that the laser damage threshold decreases as the number of defects on a surface increases and that all defects do not damage at the same fluence level. Experiments on $\mathrm{HfO}_{2} / \mathrm{SiO}_{2}$ multilayers have shown that defects with dome heights greater than $0.7 \mu \mathrm{m}$ are most susceptible to laser damage, while pit-type defects formed by the ejection of a nodule prior to illumination, do not increase damage susceptibility. 1 These two observations indicate that defect geometry may influence laser-damage susceptibility and mechanical stability of the defects. This paper tries to elucidate the relationship between the surface morphology of a nodule and its total geometry (including subsurface). The goal of this approach to defect characterization is to evaluate a defect nondestructively using a technique such as atomic force microscopy (AFM) and then irradiate the defect with laser light to determine the correlation between defect geometry and damage susceptibility.

\section{SOURCES OF SEEDS}

Various mechanisms of seed ejection from an electron-beam process are possible.6,7,8 Ejection can occur from gasses entrapped in the oxide pellets or boules. Even with adequate thermal degassing 
of the oxide source, bubbles can nucleate in the molten oxide and rise to the surface to spit out seeds. Evaporation from oxides heated above their melting temperature may cause seed ejection due to the vapor thrust breaking through the liquid surface. This molten splashing is notable during high rate evaporation, but even during low rates, localized heating variations may cause momentary splashing conditions. Localized charge build-up due to the insulating nature of dielectrics may cause micro-arc induced seed ejection. Oxides, known for their brittleness, can eject seeds by relief of thermal-induced stresses. A special case is hafnia and zirconia that have a high temperature solid-state phase transition. When these oxides crack during the phase transition, there may be accompanying seed ejection.

\section{GEOMETRIC MODEL OF NODULAR DEFECTS}

A simple model has been developed to describe the geometry of the classic parabolic nodular defect. 9 Three assumptions are made for this model: the deposition of material is omnidirectional, the seed is spherical, and the seed is much smaller than the total coating thickness. The first may be justified in that the substrates for optical coatings are generally rotated during a deposition run. ${ }^{4}$ The second should be fairly accurate if the source of the seeds is ejected liquid material. The third assumption will only be valid for smaller nodules. The layers of coating material above a seed will be concentric spherical caps. These spherical caps will intersect the planar layered structure and the resulting nodule will have parabolic cross-section. This discussion leads to the following relationships that predict the height of the nodule above the planar surface, $h$, and the surface diameter of the nodule, $D$, in terms of the seed diameter, $d$, and the seed depth, $T$.

$$
\begin{aligned}
& h=d \\
& D=(8 d T)^{1 / 2} .
\end{aligned}
$$

This model is shown in Figure 1. Besides this rather simple geometric model, quite a bit of modeling of nodular defects has been done in the past. ${ }^{10,11}$ These models used a hard disk simulation of the depositing materials and in general produced nodules of cylindrical symmetry with parabolic side wall structure, but the actual aspect ratio of the defects could be varied by changing deposition conditions in the model.

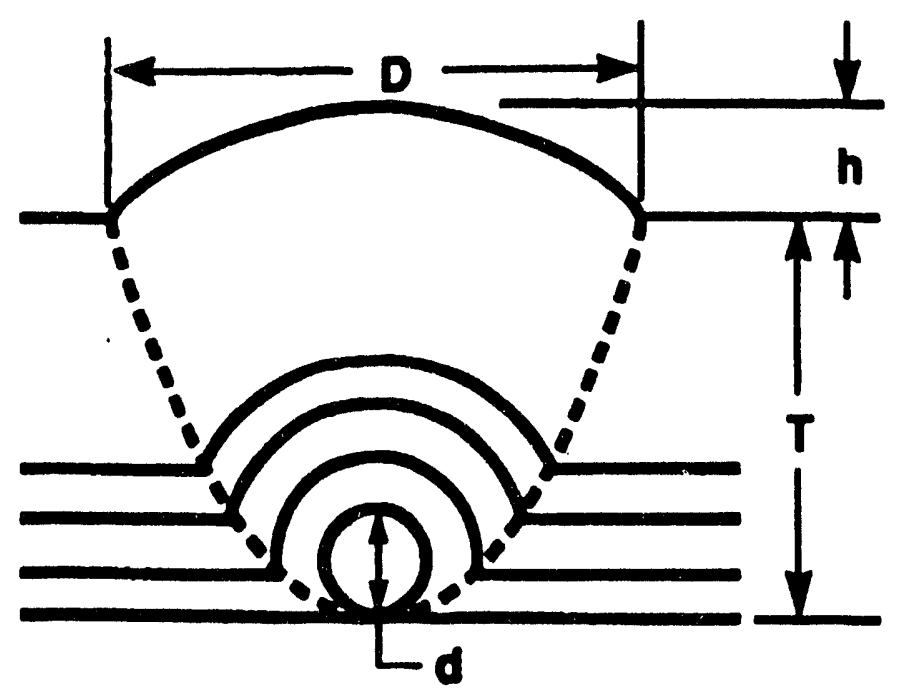


Fig. 1. Schematic diagram of nodule structure for idealized growth conditions. Dotted line is the parabolic sidewall boundary between the nodule and the multilayer.

\section{EXPERIMENT}

The sample used for this study was a normal incidence high reflector (HR) for light of wavelength $1.06 \mu \mathrm{m}$ on a $5 \mathrm{~cm}$ diameter substrate. The HR consisted of fifteen quarter wavelength layer-pairs of hafnia and silica, with a half-wave of silica as the final layer. The material was deposited using a reactive e-beam process. This sample was commercially made for use at Lawrence Livermore National Laboratory. ${ }^{12}$

To characterize the defects on the sample with multiple analytical techniques the HR was marked with an aluminum fiducial as a reference. The sample was first analyzed with a Nomarski light microscope to find an area with a high density of defects. The sample was then coated with a thin layer of metal as to make the surface conductive. A high resolution scanning electron microscope (SEM) was then used to image the defects. The defects were then scanned with an AFM to measure the true shape of the defects in three dimensions. ${ }^{13}$

The sample was then placed in a Focused Ion Beam (FIB) system to cross-section the defects. ${ }^{14}$ A FIB is essentially a SEM with a liquid-metal ion source instead of an electron source. The ion beam of this system is focused down to approximately $20 \mathrm{~nm}$. Thus, the highly accelerated and focused metal ions can sputter a surface in a well-controlled fashion such that the defects were milled in cross-section. A triangular wedge of coating was removed next to the defect such that the cross-section could be imaged. The FIB can also image because some of the ions that impinge on the surface create secondary electron that can be detected to create the image. This imaging capability allowed us to find the defects of interest. Several groups have looked at the cross-sections of defects before this study, but they created the cross-sections by cleaving the sample and hoping that a defect would be bisected. The cleaving method would not always preserve the seed that caused the defect. In addition, the same defect could not always be studied before and after cleaving. The methods used in the present work allow us to avoid the pit-falls of cleaving and mechanical cross-sectioning.

After cross-sectioning, the defects were again imaged with the high resolution of the SEM to analyze the subsurface geomeiry of the nodules. A total of ten defects of several types was characterized in this fashion. As a final step in the analysis of these defects a scanning Auger microscope (SAM) was used to identify the chemical composition of the seed material 15 of one of the nodular defects. Additional conductive material had to be deposited on the sample to obtain the required conductivity for the Auger spectroscopy to function. The SAM's spatial resolution was such that it could just image the defects and chemically analyze the seeds, but it could not analyze the layers of the coating.

\section{RESULTS}

\subsection{The "classic" nodule}

The defect shown in Fig 2 is classified as a "classic" nodule because it most closely matches the general appearance of a nodule depicted in section 3 . The nodule is cylindrically symmetric, i.e., in top view it is circular (Fig. 2 a). The transition region from the nodule's dome to the mirror's flat surface is smooth and continuous (Fig. 2 a, b, and d). The last major topographical feature of this type of nodule is that the general microstructure of the coating on and off the nodule is indistinguishable (Fig. 2 a and c). 

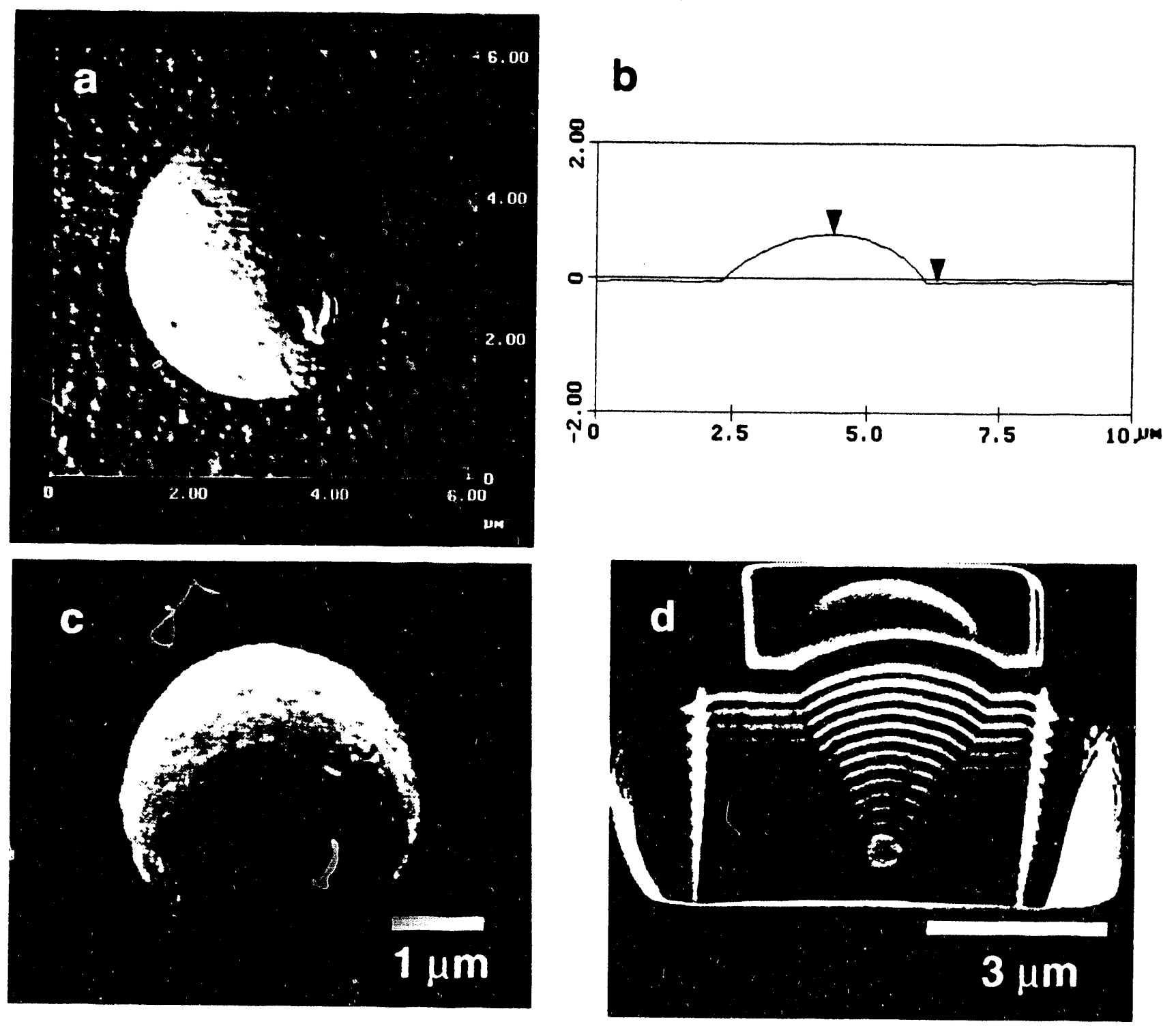

Fig. 2. The "classic" nodule. a) AFM image of nodule's surface b) Line-out of AFM image showing nodule height and transition to surface. c) SEM image of nodule. d) SEM image of FIB cross-section of nodule (note: all cross-sectional surface being imaged are at a $45^{\circ}$ angle with respect to the SEM's imaging plane, therefore the vertical dimensions of those images must be expanded by $40 \%$ ).

The FIB cross-section in Fig. $2 \mathrm{~d}$ provides the most information on the defect shape. The figure shows the shape of the seed, its depth, and the shape of the layers near the seed. The micrograph suggests that the seed is spherical as expected for a "classic" nodule, but it is actually a prolate-spheroid with its longer axis perpendicular to the surface (see Figure 2 caption). The shape of the seed suggests that it is formed by ejection of molten material that is solidified, to some extent, before impinging on the surface. The coated surface is pointing down during the deposition process so some force, possibly electro-static, must hold the seed on the substrate. 
Assuming that the seed is produced by the ejection of coating material, its composition might be deduced from the material contrast in the SEM image. The silica layers appear as the darker material in the image because the silica is less conducting than the hafnia. The half-wave over-coat of silica is clearly visible in the image. The brighter layers then must be the hafnia. The seed shows similar contrast to the hafnia layers and can therefore be assumed to be hafnia. Auger analysis described below confirms this assumption. Note that the seed was deposited at the end of the third layer of hafnia. Such information can be important to the operator of the coating apparatus, because it might be correlated to a particular deposition event that causes seed ejection.

The structure of the layers deposited above the seed can be gained next from this image (Fig. $2 \mathrm{~d}$ ). The structure of the boundary region between the defect and the surrounding layers changes as the deposition progresses. Close to the seed this boundary region is convoluted and the individual layers are not continuous. But, as more material is deposited the boundary heals and the layers become continuous.

\subsection{The "non-classic" nodule}

"Non-classic" nodule is used to describe the defect in Fig. 3 because it retains some of the properties of the "classic" while losing others. The nodule is cylindrically symmetric, i.e. in top view it is circular (Fig. 3 a), like the "classic." However, in this case the edges of the nodule are very rough and the transition is rough from the nodule to the surface. In addition, the coating surface dips next to the edge of the nodule (Fig. $3 \mathrm{~b}$ and d), which is most likely caused by the larger seed dimensions creating a shadow region next to the defect. As with the "classic" nodule the general microstructure of the coating on and off the nodule is very similar (Fig. $3 \mathrm{a}$ and $\mathrm{c}$ ).

The most information can again be gained from the FIB cross-section in Fig $3 \mathrm{~d}$. The seed in this defect is much closer to spherical than the seed in the "classic" defect in Fig. 2. The main difference, however, is that the seed in Fig. 3 is much larger than that in Fig. 2. It again appears to be a hafnia seed. This seed was deposited some time after the third layer of the coating run. An extremely convoluted boundary region is present at this nodule. It also appears to have voids in this region (voids are basically indistinguishable from insulators to the SEM). The profile view shows how the coating dips next to the defect and in this case the convoluted boundary region never heals to produce continuous layers.

\subsection{The complex nodule}

In this case a very large non-uniform seed produced a very complex nodule structure (Fig. 4). Several observations can be made by analyzing the AFM image in Fig. 4a: the edges of the nodule are very rough in some areas, the coating dips next to the defect, and the defect is non-circular leading to the assumption that the seed is not a sphere. When the sample was removed from the FIB vacuum chamber to the SEM, the cross-s ctioned fell apart, without any direct force being annlied, (Fig. $4 \mathrm{~b}$ ).

\subsection{The pit}

Another type of defect found on this sample was a pit resulting from the defects falling off the surface after the deposition was completed (Fig. 5). These pit-type defects were measured to have a variety of sizes and depths. As can be seen in Fig. 5, this pit was formed by a very large extended object that shadowed the edges such that a noticeable dip in the coating layers can be seen next to the pit itself. The seed that initiated this pit remained on the surface throughout the deposition of the coating and fell off after all layers were deposited. This was a general observation of all the pits seen in this study, i.e. none of the pits were back filled with coating material. 


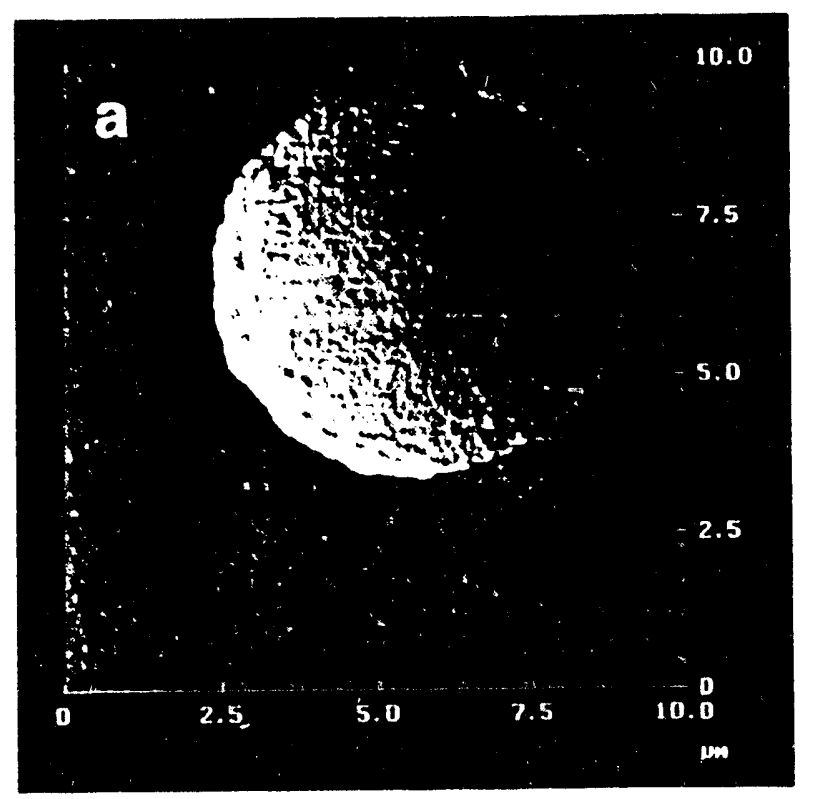

b
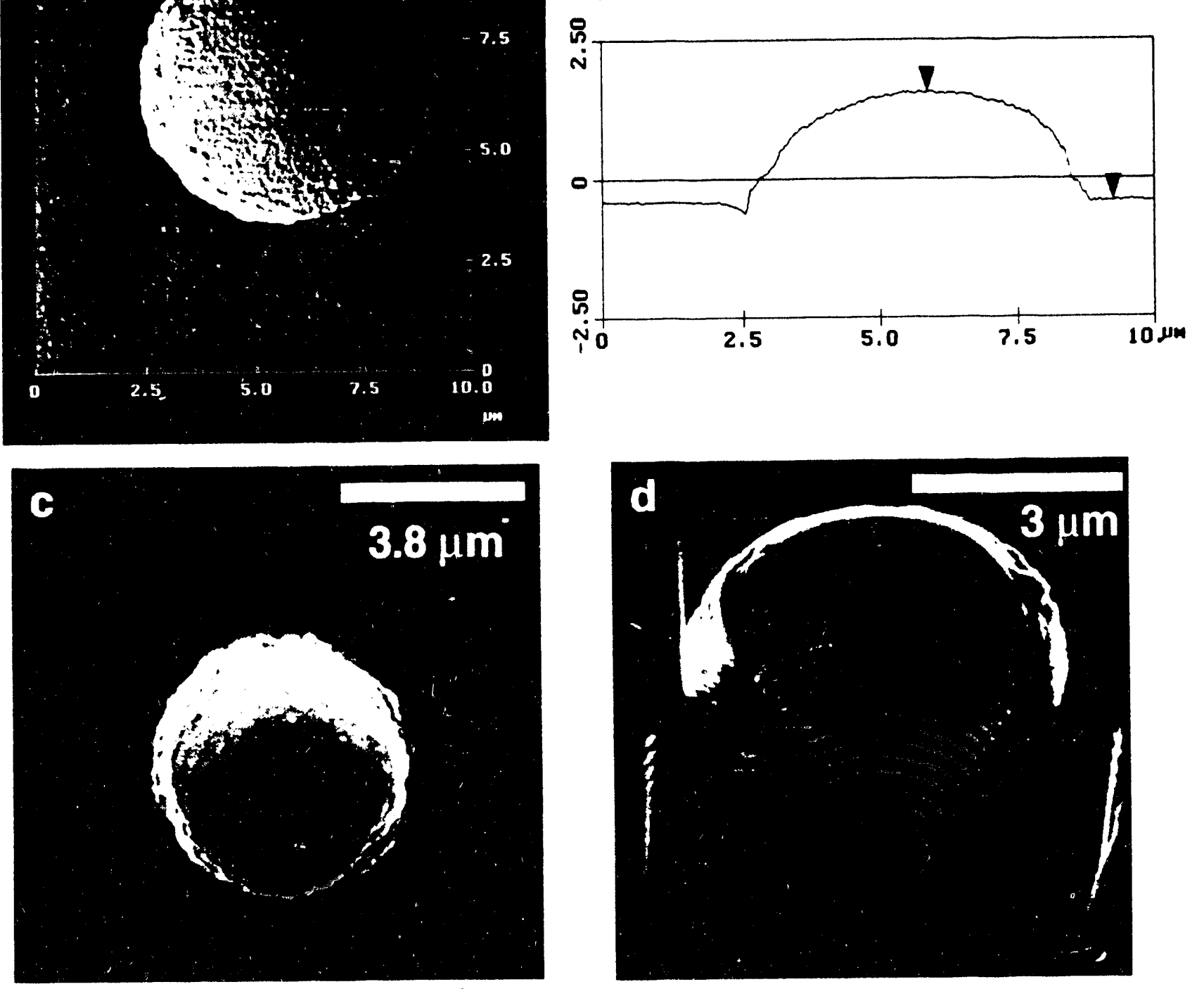

Fig. 3. The "non-classic" nodule. a) AFM image of nodule's surface b) Line-out of AFM image showing nodule height and transition to surface. c) SEM image of nodule. d) SEM image of FIB cross-section of nodule. 

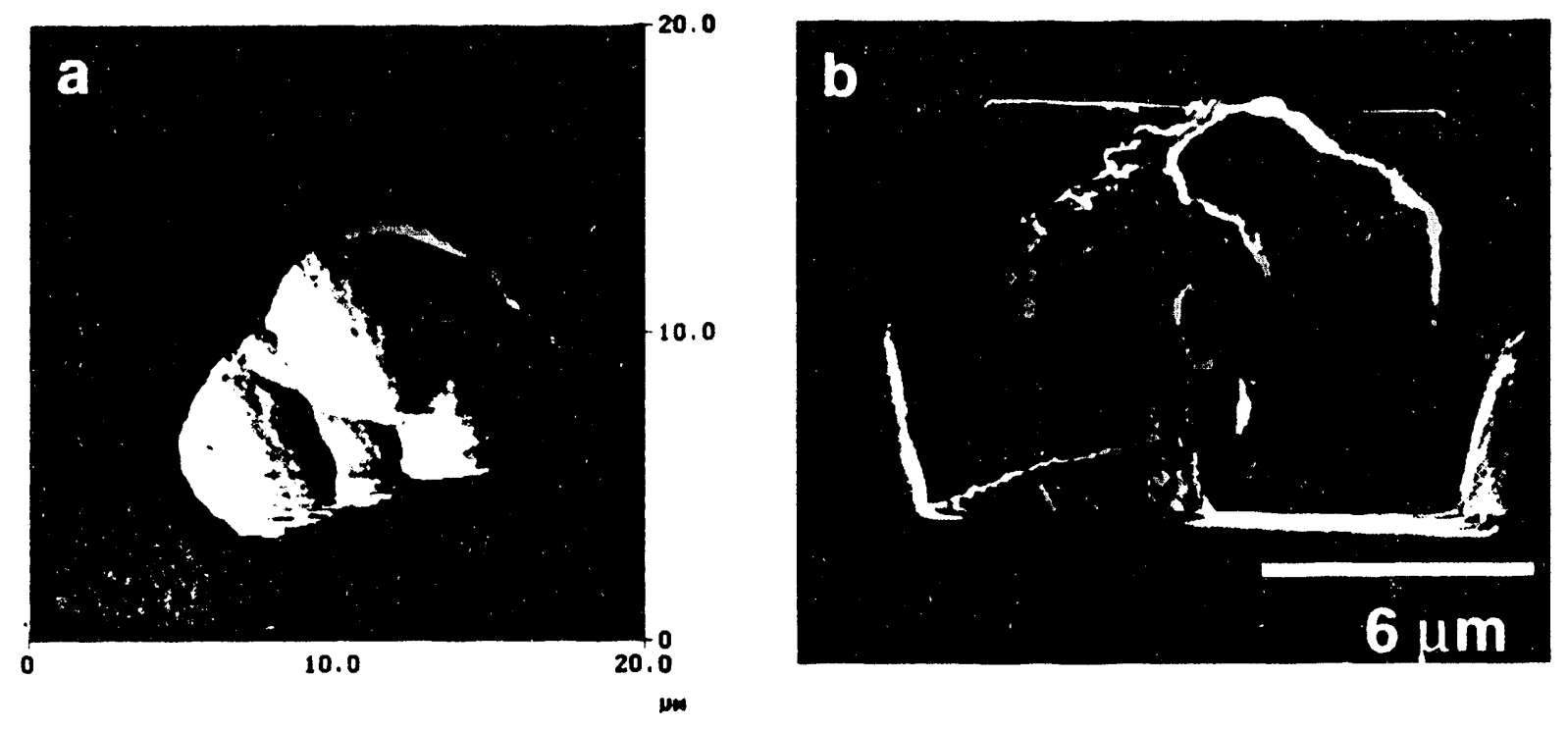

Fig. 4. The complex nodule. a) AFM image of nodule's surface b) SEM image of FIB cross-section of nodule.

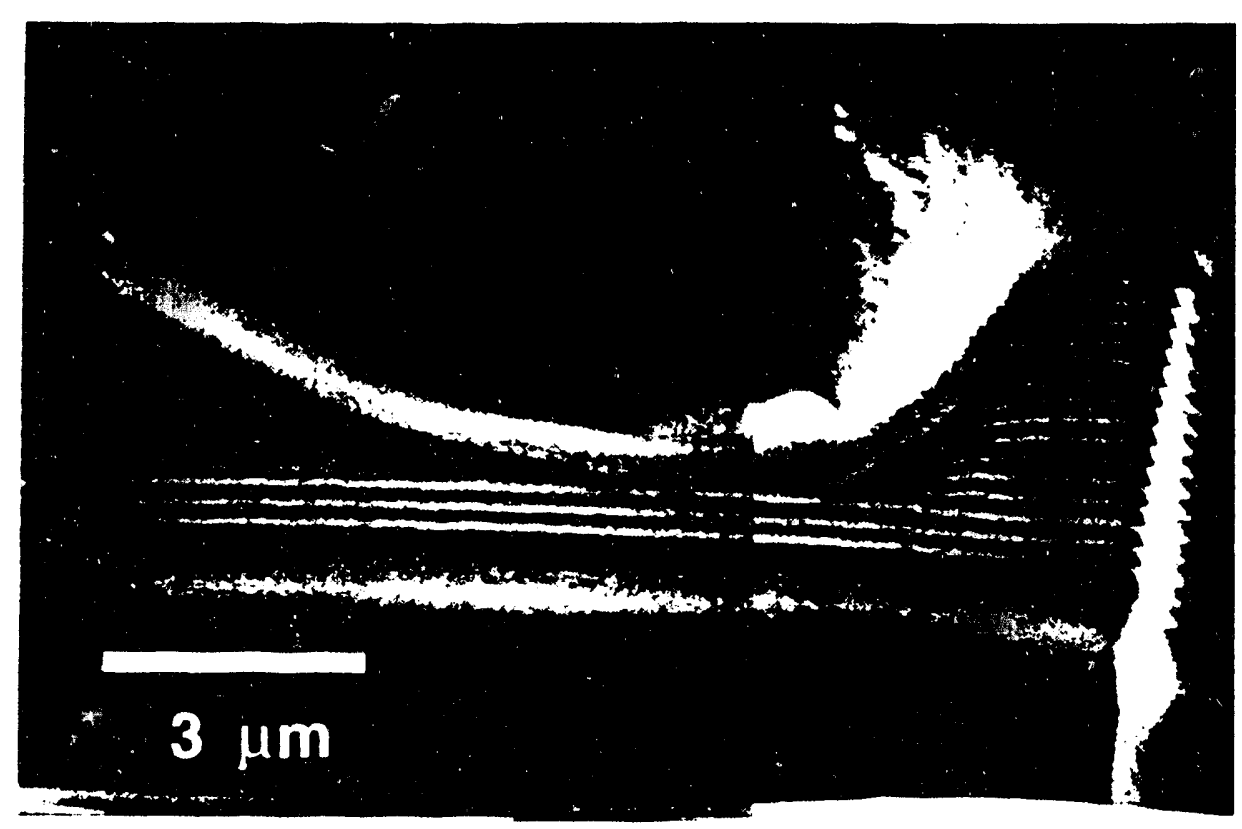

Fig. 5. The pit. SEM image of FIB cross-section of defect. 


\subsection{Seed composition}

The sample was analyzed using scanning Auger microscopy (SAM) to identify the chemical constituents of the seed. The Auger spectrum of the seed is shown in Fig. 6. The spatial resolution of the SAM was not high enough to resolve the layers of the coating, but it was capable of resolving the seed itself. The analysis shows the presence of hafnium and oxygen. There is a peak that suggests silicon in the seed, but upon analysis of high energy Auger peaks (between 1400 and $1700 \mathrm{eV}$ ) the presence of silicon is ruled out.

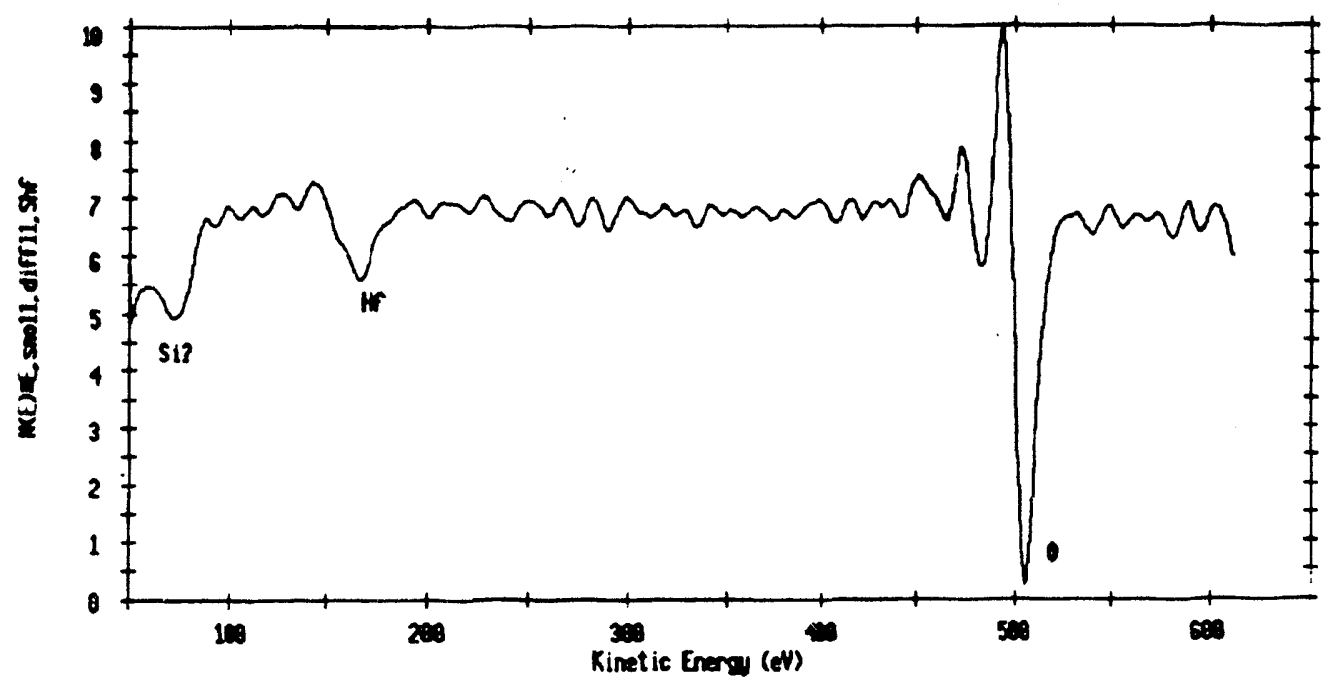

Fig. 6. Auger electron spectrum of defect seed.

\section{DISCUSSION}

Because the nodule dimensions can be accurately measured from the images provided, a comparison between actual nodule geometry and the model discussed in section 4 can be made. The model first predicts that the height of the nodule and the diameter of the seed (actually the height of the seed is needed because the seed is not spherical) are equal. As can be seen in Fig. 7 a there is strong agreement between the seed height and the nodule height for the four nodules examined $(0.93$ degree of fit). Figure $7 \mathrm{~b}$ checks the validity of equation (1) by plotting the nodule diameter squared versus the product of the seed size and depth. In this case the data (square dots) does show a linear relationship but the slope is less than half of the predicted value (3.2 versus a theoretical value of 8 ). The assumption of omnidirectional deposition is probably false leading to this discrepancy. If some contribution of unidirectional deposition is assumed, the lower aspect ratio $(\mathrm{D} / \mathrm{h})$ nodules observed in this work might be explained. The exact relationship between seed and nodule dimensions is likely different for each deposition chamber and method. This relationship, however, can be determined experimentally as outlined in this work. 

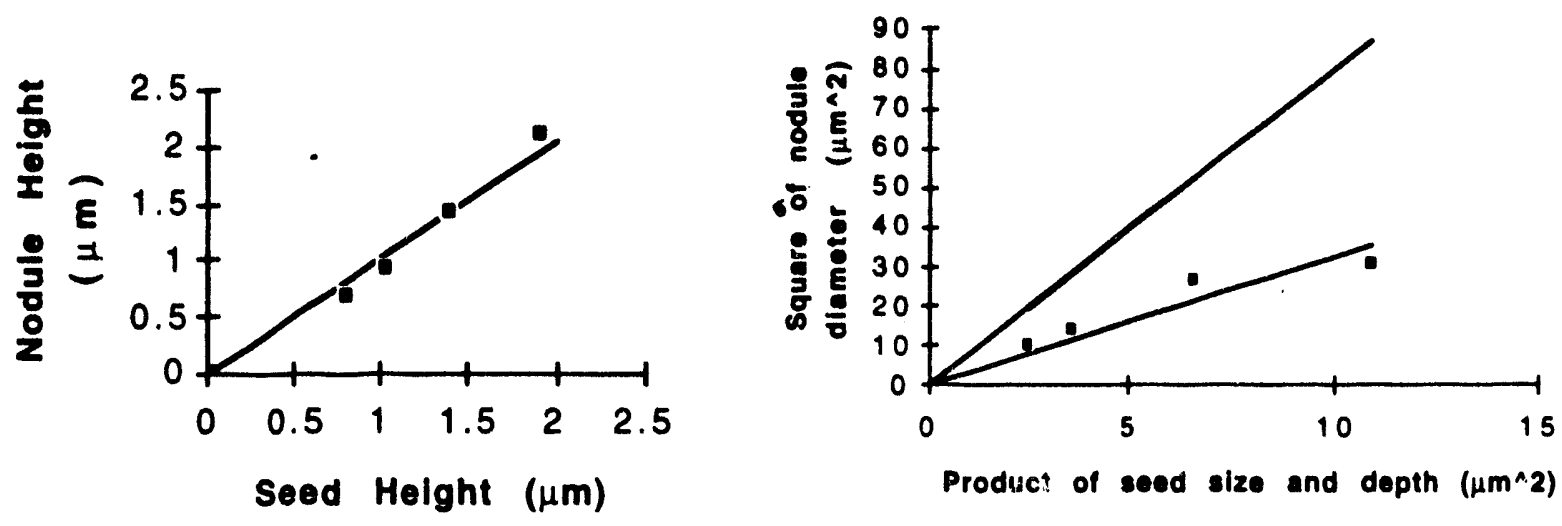

Fig. 7. Measured vs. predicted defect geometries. a) Nodule vs. seed height b) Square of nodule diameter vs. seed size and depth.

In addition to showing that surface dimensions measured using the AFM provided some insight into underlying defect shape, we also showed that AFM measurements could provide some insight into the nodule/coating boundary region. Take for example the nodules in Fig. 3 and Fig. 2. A smooth nodule-to-surface transition region, as measured by the AFM, is associated with smooth continuous layers below the surface. In contrast, a rough nodule with a dip in the coating at the nodule boundary is correlated with a very convoluted sub-surface boundary region. An important implication of this analysis is the relative mechanical stabilities of different nodules can be surmised based on surface topography measurements. Nodules with small and/or very deep seeds form classic nodules. Therefore they have smooth boundaries, continuous layers, and are expected to be somewhat mechanically stable. Nodules with large and/or shallow seeds, on the other hand, are less classic with rough boundary regions and voids, and are likely mechanically less stable.

Considering the work of Staggs et. al. ${ }^{5}$ where taller nodules damage at relatively lower laser fluences, and previous E-field modeling work, showing larger and shallower defects couple more strongly with laser light, ${ }^{16}$ the mechanical stability issues found here are likely a contributing factor to the nodule's laser damage susceptibility. In other words, high dome nodules are caused by large seeds or shallow seeds. These defects have discontinuous boundaries to the multilayer. The discontinuity is a measure of the mechanical stability of the nodule.

Low fluence laser conditioning has been shown to lower the damage susceptibility of the nodules. One possible damage/conditioning mechanism of nodules is the interaction of the laser light with the voids in the nodule's sub-surface boundary region. It has been shown that the presence of smooth voids at nodule boundaries has little influence on local E-fields ${ }^{16}$ so the closing of these voids due to laser conditioning is not likely to influence the subsequent damage susceptibility. Surface smoothing observed using the $\mathrm{AFM}^{5}$ indicates that the conditioning process may fuse the nodule into the coating and therefore provide mechanical stability. Staggs et. al. 5 also showed that pits do not influence the damage susceptibility. Therefore, if i: the conditioning process the large nodules are popped out leaving smooth pits, the coating would also be resistant to further damage. It is likely that one or both of these conditioning methods are taking place in practice. 


\section{CONCLUSIONS}

A nodule's underlying structure can be inferred from AFM images of the nodule's surface topography. Once the defect's structure is determined, its relative mechanical stability can be inferred based on the cross-section images presented in this work. Nodules with large (and shallow) seeds have boundary regions that are discontinuous from the seed to the surface of the film and are therefore weakly bonded to the film. Nodules with small (and deep) seeds have boundary regions that are discontinuous initially but become continuous near the surface of the film and are therefore strongly bonded to the film.

By AES studies, the seeds of the nodules in the hafnia/silica multilayers studied here are generated by the hafnia source. Therefore, to eliminate nodular defects, a method of depositing hafnia without particle ejection from the source in the reactive e-beam process must be found.

From this work on nodule cross-sectioning and prior laser damage studies two possible mechanisms for laser conditioning can be proposed: either the nodules pop out non-destructively or the nodules are welded to the coating such that they cannot pop out.

\section{ACKNOWLEDGMENTS}

This work was performed under the auspices of the US Department of Energy by Lawrence Livermore National Laboratory under contract number W-7405-ENG-48.

\section{REFERENCES}

1 M. C. Staggs, M. R. Kozlowski, W. J. Siekhaus, and M. Balooch, "Correlation of damage threshold and surface geometry of nodular defects in hr coatings as determined by in-situ atomic force microscopy," Laser-Induced Damage in Optical Materials: 1992, SPIE Vol. 1884, pp. 234-242, 1993.

2 L. F. Johnson, E. J. Ashley, T. M Donovar, !. B. Franck, R. W. Woolever, and R. Z. Dalbey, "Scanning electron microscopy studies of laser damage initiating defects in $\mathrm{ZnSe} / \mathrm{ThF} 4$ and $\mathrm{SiH} / \mathrm{SiO}_{2}$ multilayer coatings." Laser Damage in Optical Materials: 1984, NBS Special Pub. 727, p. 356, 1984.

3 M. R. Kozlowski, M. C. Staggs, M. Balooch, R. J. Tench, and W. J. Siekhaus, "The surface morphology of as-deposited and laser-damaged dielectric mirror coatings studied in-situ by atomic force microscopy," Scanning Microscopy Instrumentation: 1991, SPIE Vol. 1556, pp. 68-78, 1992.

4 B. Liao, D. J. Smith, and B McIntyre, "The formation and development of nodular defects in optical coatings," Laser-Induced Damage in Optical Materials: 1985, NBS Special Pub. 746, pp. $305-$ 318, 1985.

5 M. C. Staggs, M. Balooch, M. R. Kozlowski, and W. J. Siekhaus, "In Situ Atomic Force Microscopy of laser-conditioned and laser-damaged $\mathrm{HfO} 2 / \mathrm{SiO} 2$ dielectric mirror coatings," Laserinduced Damage in Optical Materials: 1991. SPIE Vol. 1624, pp. 375-385. 1992 ,

6 M. R. Kozlowski, and R. Chow, "The role of defects in laser damage of multilayer coatings," Laser-induced Damage in Optical Materials: 1991 SPIE this proceedings Vol., 1994.

7 S. Schiller, M. Neumann, H. Morgner, and N. .Schiller, "Progress in high-rate electron beam evaporation of oxides for web coating, "in the $36 \mathrm{t}^{\mathrm{h}}$ Annual Technical Conference Proceedings of the Society of Vacuum Coaters, eds. , April, 1993 , Dallas, TX, pp. 278-292, 1993.

8 R. Chow, S. Falabella, G. E. Loomis, F. Rainer, C. J. Stolz, M. R. Kozlowski, "Absorption and damage thresholds of low-defect density hafnia deposited with activated oxygen," Laser-induced Damage in Optical Materials: 1992, SPIE vol. 1848, pp. 349-360, 1993.

9 S. A. Letts, D. W. Myers, and L. A. Witt, "Ultrasmooth plasma polymerized coatings for laser fusion targets," J. Vac. Sci Tech., Vol. 19(3), pp. 739-742, 1981. 
10 K. H. Guenther, "Microstructure of vapor-deposited optical coating," Applied Optics, Vol. 23 No. 21, PP. 3806-3816, 1984, and references within.

11 D. J. Smith, "Modeling of nodular defects in thin films for various deposition techniques," Modeling of Optical Thin Films SPIE Vol. 821, pp. 120-128, 1987, and references within.

12 Optical Coatings Laboratory Incorporated, Santa Rosa, CA.

13 AFM produced by Digital Instruments Inc., Santa Barbara, CA.

14 Work performed at FIB Applied Semiconductor Technology, San Jose, CA.

15 Work performed at Charles Evans \& Associates, Redwood City, CA.

16 J. F. DeFord, and M. R. Kozlowski, "Modeling of electric-field enhancement at nodular defects in dielectric mirror coatings." Laser-induced Damage in Optical Materials: 1992, SPIE vol. 1848, pp. 455-470, 1993. 


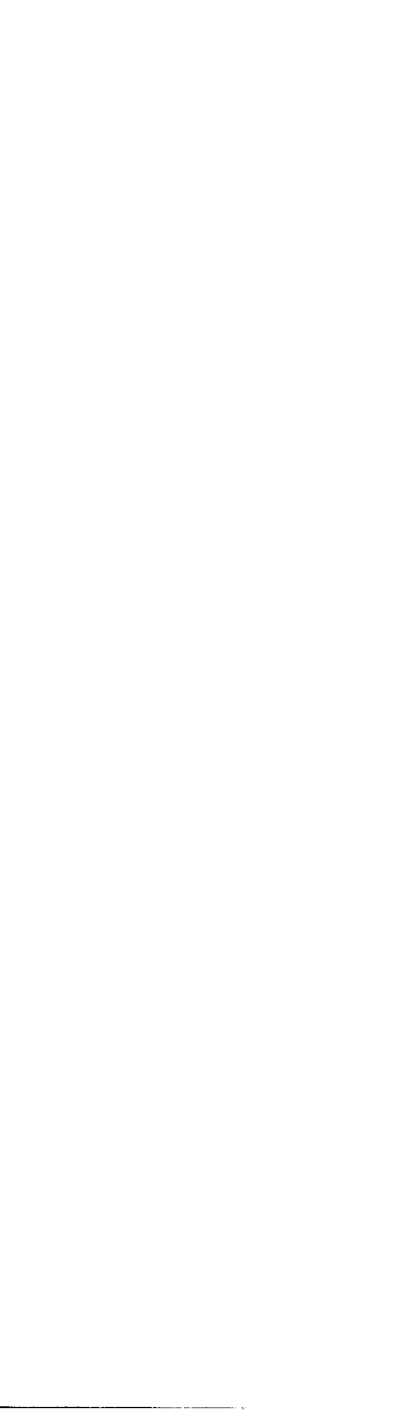

